\title{
(ص) Simplifying the use of prognostic information in traumatic brain injury. Part 1: The GCS-Pupils score: an extended index of clinical severity
}

\author{
Paul M. Brennan, MBBChir, FRCS, PhD, ${ }^{1}$ Gordon D. Murray, MA, PhD, ${ }^{2}$ and \\ Graham M. Teasdale, MBBS, FRCP, FRCS ${ }^{3}$ \\ ${ }^{1}$ Centre for Clinical Brain Sciences and 2 Usher Institute of Population Health Sciences and Informatics, University of Edinburgh; \\ and ${ }^{3}$ Institute of Health and Wellbeing, University of Glasgow, United Kingdom
}

\begin{abstract}
OBJECTIVE Glasgow Coma Scale (GCS) scores and pupil responses are key indicators of the severity of traumatic brain damage. The aim of this study was to determine what information would be gained by combining these indicators into a single index and to explore the merits of different ways of achieving this.
\end{abstract}

METHODS Information about early GCS scores, pupil responses, late outcomes on the Glasgow Outcome Scale, and mortality were obtained at the individual patient level by reviewing data from the CRASH (Corticosteroid Randomisation After Significant Head Injury; $n=9,045$ ) study and the IMPACT (International Mission for Prognosis and Clinical Trials in $\mathrm{TBI} ; \mathrm{n}=6855$ ) database. These data were combined into a pooled data set for the main analysis.

Methods of combining the Glasgow Coma Scale and pupil response data varied in complexity from using a simple arithmetic score (GCS score [range 3-15] minus the number of nonreacting pupils [0,1, or 2]), which we call the GCS-Pupils score (GCS-P; range 1-15), to treating each factor as a separate categorical variable. The content of information about patient outcome in each of these models was evaluated using Nagelkerke's $R^{2}$.

RESULTS Separately, the GCS score and pupil response were each related to outcome. Adding information about the pupil response to the GCS score increased the information yield. The performance of the simple GCS-P was similar to the performance of more complex methods of evaluating traumatic brain damage. The relationship between decreases in the GCS-P and deteriorating outcome was seen across the complete range of possible scores. The additional 2 lowest points offered by the GCS-Pupils scale (GCS-P 1 and 2) extended the information about injury severity from a mortality rate of $51 \%$ and an unfavorable outcome rate of $70 \%$ at GCS score 3 to a mortality rate of $74 \%$ and an unfavorable outcome rate of $90 \%$ at GCS-P 1 . The paradoxical finding that GCS score 4 was associated with a worse outcome than GCS score 3 was not seen when using the GCS-P.

CONCLUSIONS A simple arithmetic combination of the GCS score and pupillary response, the GCS-P, extends the information provided about patient outcome to an extent comparable to that obtained using more complex methods. The greater range of injury severities that are identified and the smoothness of the stepwise pattern of outcomes across the range of scores may be useful in evaluating individual patients and identifying patient subgroups. The GCS-P may be a useful platform onto which information about other key prognostic features can be added in a simple format likely to be useful in clinical practice.

https://thejns.org/doi/abs/10.3171/2017.12.JNS172780

KEYWORDS Glasgow Coma Scale; head injury; traumatic brain injury; trauma; prognosis; pupil reactivity

ABBREVIATIONS CRASH = Corticosteroid Randomisation After Significant Head Injury; GCS = Glasgow Coma Scale; GCS-P = GCS-Pupils score; GOS = Glasgow Outcome Scale; IMPACT = International Mission for Prognosis and Clinical Trials in Traumatic Brain Injury; PRS = pupil reactivity score; TBI = traumatic brain injury. SUBMITTED November 3, 2017. ACCEPTED December 23, 2017.

INCLUDE WHEN CITING Published online April 10, 2018; DOI: 10.3171/2017.12.JNS172780. 
A SSESSMENT of a patient's clinical condition, and how it may change, is a cornerstone of care for people with head injury and for those with other kinds of acute brain damage. The Glasgow Coma Scale (GCS) is widely used for this purpose, reflecting its utility in observing a patient's responsiveness, or so-called "consciousness level," and communicating this finding clearly and consistently. ${ }^{22,29,31}$ Decisions about case management are also influenced by perceptions of the relationship between a patient's early condition and likely outcome.

Although estimates of prognosis are best made using mathematical methods that combine information about multiple aspects of the patient's condition, these have not found widespread acceptance in clinical practice. ${ }^{26}$ Instead, simple scoring systems for stratifying injury severity appeal to clinicians in every field of medicine. ${ }^{25}$ These trade off a loss of detail and specificity of information for the ease and transparency of calculation of the score.

The Glasgow Coma Scale is used to assess 3 aspects of a patient's responsiveness (eye, verbal, and motor responses). Each of these aspects contains information about prognosis..$^{21,30}$ Soon after the Glasgow Coma Scale was described, the findings of the 3 components were combined in a summary total score, derived from the simple addition of a notation assigned to each of its components. ${ }^{28}$ The GCS score is widely used as an index of "overall" brain damage, which is the most important feature in distinguishing head injuries of differing severities and in monitoring patient progress and estimating prognosis. ${ }^{5,29}$ In accord with this, focal indices, such as brainstem features, were not incorporated into the scale and were intended to be assessed separately. ${ }^{7}$ Nevertheless, some clinicians have held the view that more complex scores, which would include extra physiological features, would nevertheless be useful. 2,3,32

The GCS score, together with information about pupil reaction, conveys to the physician most of the clinical predictive information in head-injured patients. ${ }^{4,10,27}$ In the present study we investigated various ways of combining information gained from these two key features into an index of prognosis that would cover either mortality or unfavorable outcome (vegetative state or severe disability) in acute head-injured patients. Our aims were to determine the yield provided by various methods of combining information about these two features and comparing the merits of a simple, transparent arithmetic combination, which would likely be applied easily, with the merits of more complex models.

\section{Methods}

Our investigations centered on information obtained from 2 large head-injury databases: CRASH (Corticosteroid Randomisation After Significant Head Injury) ${ }^{23}$ and IMPACT (International Mission for Prognosis and Clinical Trials in TBI). ${ }^{10}$ In the CRASH study 10,008 adults with head injury were recruited from 239 hospitals in 49 countries. The IMPACT database, on the other hand, contains data on 11,989 patients with traumatic brain injury (TBI), which were collected prospectively for 11 different studies including 8 randomized controlled trials and 3 epidemiological studies.

\section{GCS Score and Pupil Reactivity}

From the CRASH and IMPACT databases we identified patients for whom both GCS score and pupil reactivity information were available. In the CRASH study, the eye, verbal, and motor components of the Glasgow Coma Scale were recorded for each patient at the time of randomization; from these data GCS total scores were calculated. In each of the 11 studies included in the IMPACT study, GCS scores were determined at different time periods. We used the same data as the IMPACT model; this model was developed using a "derived GCS score," defined as the GCS score assigned to the patient closest to randomization or upon entry into the study. ${ }^{10}$ In both studies pupil reaction to light was coded separately for each eye and was recorded at the same time as the assessment of the GCS score.

\section{Patient Outcome}

The proportions of patients in each study who either were dead or had an unfavorable outcome (vegetative state or severe disability) according to the Glasgow Outcome Scale (GOS) were determined. In the CRASH study, patient outcome was assessed by applying the GOS 6 months after injury by using a structured postal questionnaire; outcome information was available for $97 \%$ of patients. ${ }^{23}$ In the IMPACT database, patient outcome was also determined by applying the GOS classification 6 months after head injury. Here the GOS data were available for 11,384 patients (95\%); in cases in which outcome data at the 6-month time point were unavailable, data obtained 3 months after head injury were used..$^{10}$

\section{Combining Information About GCS Score and Pupil Reactivity}

We examined the relationships between the GCS total score and pupil reaction to light and between the interactions of these factors and outcome 6 months after injury. Data from the CRASH study, the IMPACT database, and also from a pooled data set were examined. Contingency tables and graphic plots of the associations in data between GCS score, pupil reaction, and patient outcomes were inspected.

We developed a method to combine a patient's GCS score and pupil findings into a single, unidimensional index. We first constructed a pupil reactivity score (PRS) to reflect the number of nonreactive pupils. If both pupils were unreactive to light the score was 2 . If only 1 pupil was unreactive to light the score was 1 . If neither pupil was unreactive to light the score was 0 . Next, a combined GCS-Pupils score (GCS-P) was obtained simply by subtracting the PRS from the GCS total score: GCS-P = GCS score - PRS. Since a GCS total score can range from 3 to 15 , a GCS-P thus has a range of possible values from 1 to 15 .

\section{Information Content}

We examined the extent to which various ways of 
combining information about the GCS score and pupil reactivity influenced information provided about patient outcome. Our aim was to discover if using the GCS-P as a simple arithmetic index substantially influenced the information provided about the severity of a patient's prognosis-as compared either to its components alone or to more complex methods of combination.

We used logistic regression to model patient outcome, using the following methods in decreasing order of complexity:

a. treating the GCS score and the number of nonreactive pupils separately as categorical variables (expected to be the best performing model);

b. treating the GCS score as a linear variable and keeping the number of nonreactive pupils as a categorical variable;

c. treating the GCS score as a categorical variable and treating the number of nonreactive pupils as a linear variable;

d. treating both the GCS score and the number of nonreactive pupils as separate linear variables;

e. treating the GCS-P as a categorical variable; and

f. treating the GCS-P as a linear variable.

Models $\mathrm{b}$ and $\mathrm{c}$ are simplifications of model $\mathrm{a}$, and model $\mathrm{d}$ is a simplification of models $\mathrm{b}$ and $\mathrm{c}$. In addition, various models were fitted using only one variable: GCS score or the number of nonreacting pupils.

The proportion of variability in outcome "explained" by each model was assessed using Nagelkerke's $\mathrm{R}^{2}{ }^{15}$

\section{Results}

Complete data (GCS score, pupil reactivity, and outcome data) were available for 15,900 of the 21,997 patients whose information was contained in the two databases: $90 \%$ of patients in the CRASH study but only $57 \%$ of patients in the IMPACT database (Table 1). In the IMPACT database, the low proportion of patients for whom a complete data set containing GCS score, pupil reactivity, and outcome data were available reflects the fact that the data were assembled retrospectively from multiple smaller studies. In contrast, the CRASH data came from a single, prospectively conducted randomized controlled trial.

Reflecting the focus of its component studies, most patients whose data were found in the IMPACT database had a severe injury, but in the CRASH study the proportions of patients with a severe, moderate, or mild injury were similar (30.4\%-39.0\%). In the CRASH study the criteria for recruitment specified a GCS score of 14 or less, and thus there were few patients (10 [0.1\%]) assigned a GCS score of 15; in the IMPACT database there was no such criterion, and there were 318 patients (4.6\%) with GCS score 15 . Pupil reactivity was lost unilaterally in $6.2 \%$ and bilaterally in $8.8 \%$ of patients in the CRASH study, and unilaterally in $12.2 \%$ and bilaterally in $18.9 \%$ of patients in the IMPACT database.

Despite these overall differences, in each study a similar proportion of patients had died by 6 months after injury $(24.0 \%$ of patients mentioned in the IMPACT database and $23.7 \%$ in the CRASH study). More cases included in
TABLE 1. Principal patient characteristics

\begin{tabular}{|c|c|c|c|}
\hline Factor & $\begin{array}{l}\text { CRASH } \\
\text { Database }\end{array}$ & $\begin{array}{l}\text { IMPACT } \\
\text { Database }\end{array}$ & $\begin{array}{l}\text { Pooled } \\
\text { Data }\end{array}$ \\
\hline Patients enrolled in study & 10,008 & 11,989 & 21,997 \\
\hline $\begin{array}{l}\text { Patients for whom GCS, } \\
\text { pupil, \& outcome data } \\
\text { are all available (\% of } \\
\text { patients enrolled in study) }\end{array}$ & $9045(90)$ & $6855(57)$ & $15,900(72)$ \\
\hline Mean age in yrs (SD) & $37.1(17.0)$ & $33.9(16.3)$ & $35.7(16.8)$ \\
\hline $\mathrm{M} / \mathrm{F}$ ratio & $81: 19$ & $77: 23$ & $79: 21$ \\
\hline \multicolumn{4}{|l|}{ GCS score } \\
\hline 13-15 (mild head injury) & $2769(30.6)$ & $546(8.0)$ & $3315(20.8)$ \\
\hline $\begin{array}{l}\text { 9-12 (moderate head } \\
\text { injury) }\end{array}$ & $2752(30.4)$ & 776 (11.3) & $3528(22.2)$ \\
\hline 3-8 (severe head injury) & $3524(39.0)$ & $5533(80.7)$ & $9057(57.0)$ \\
\hline \multicolumn{4}{|l|}{ No. of reactive pupils } \\
\hline 0 & 799 (8.8) & 1294 (18.9) & $2093(13.2)$ \\
\hline 1 & $564(6.2)$ & $837(12.2)$ & $1401(8.8)$ \\
\hline 2 & $7682(84.9)$ & $4724(68.9)$ & $12,406(78.0)$ \\
\hline Mortality ( $\%$ of patients) & 23.7 & 24.0 & 23.8 \\
\hline $\begin{array}{l}\text { Unfavorable outcome (\% of } \\
\text { patients) }\end{array}$ & 36.4 & 45.0 & 40.1 \\
\hline
\end{tabular}

Values represent number of patients (\%) for whom relevant data are available.

the IMPACT database had an unfavorable outcome (death, vegetative state, or severe disability): $45.0 \%$ of patients compared with $36.4 \%$ in the CRASH study.

To counterbalance variations in recruitment criteria and consequent data input and outcome characteristics, to increase generalizability of the present study findings, and to increase patient sample size, data from the two sources were combined into a pooled data set. Unless specified otherwise, this pooled data set is the source of results presented in this paper. Separate detailed information for each data set are available as supplementary data.

\section{Relation of GCS Score and Pupil Response to Patient Outcome}

Declines in GCS scores were associated with increasing rates of mortality and unfavorable outcome in each of the CRASH and IMPACT data sets (Supplementary Table $1 \mathrm{a}$ and $\mathrm{b}$ ). In the pooled data the patient mortality rate was $51 \%$ at GCS score 3 and 54\% at GCS score 4; after that the mortality rate declined progressively to $3 \%$ at GCS score 15 (Table 2). There was a similar relationship between the GCS score and unfavorable outcome at 6 months postinjury (Table 3), with a decline in the rate of unfavorable outcome from $70 \%$ at GCS score 3 to $12 \%$ at GCS score 15. Again, the proportion of patients experiencing an unfavorable outcome was higher at GCS score 4 (79\%) than at GCS score 3 (70\%). These patterns were also reflected in an association between patients' loss of pupil reactivity and worse outcome in each data set. In the pooled data, overall mortality rose from $16 \%$ when both pupils reacted, to $38 \%$ when only one reacted, and to $59 \%$ when neither pupil reacted. In a similar way, unfavorable outcome rose 
TABLE 2. Patients with a TBI stratified by GCS score and number of reactive pupils, and the percentage of these patients who were dead 6 months after injury

\begin{tabular}{|c|c|c|c|c|c|c|c|c|}
\hline \multirow[b]{3}{*}{ GCS Score } & \multicolumn{6}{|c|}{ No. of Reactive Pupils } & & \\
\hline & \multicolumn{2}{|c|}{0} & \multicolumn{2}{|c|}{1} & \multicolumn{2}{|c|}{2} & \multicolumn{2}{|c|}{ Total No. of Patients } \\
\hline & $\begin{array}{c}\text { No. of } \\
\text { Patients }\end{array}$ & $\begin{array}{c}\% \text { Dead at } \\
6 \text { Mos }\end{array}$ & $\begin{array}{c}\text { No. of } \\
\text { Patients }\end{array}$ & $\begin{array}{c}\% \text { Dead at } \\
6 \text { Mos }\end{array}$ & $\begin{array}{c}\text { No. of } \\
\text { Patients }\end{array}$ & $\begin{array}{c}\% \text { Dead at } \\
6 \text { Mos }\end{array}$ & $\begin{array}{c}\text { No. of } \\
\text { Patients }\end{array}$ & $\begin{array}{c}\% \text { Dead at } \\
6 \text { Mos }\end{array}$ \\
\hline 3 & 634 & 74.45 & 178 & 47.19 & 617 & 28.04 & 1429 & 51.01 \\
\hline 4 & 458 & 71.40 & 239 & 56.49 & 462 & 36.58 & 1159 & 54.44 \\
\hline 5 & 322 & 54.04 & 228 & 40.35 & 673 & 30.91 & 1223 & 38.76 \\
\hline 6 & 326 & 42.94 & 259 & 37.07 & 1278 & 23.55 & 1863 & 28.82 \\
\hline 7 & 204 & 32.35 & 208 & 28.37 & 1792 & 18.75 & 2204 & 20.92 \\
\hline 8 & 64 & 42.19 & 111 & 24.32 & 1004 & 19.22 & 1179 & 20.94 \\
\hline 9 & 27 & 25.93 & 46 & 32.61 & 711 & 18.00 & 784 & 19.13 \\
\hline 10 & 23 & 30.43 & 29 & 37.93 & 788 & 17.13 & 840 & 18.21 \\
\hline 11 & 12 & 16.67 & 28 & 25.00 & 836 & 11.60 & 876 & 12.10 \\
\hline 12 & 8 & 12.50 & 22 & 9.09 & 998 & 9.12 & 1028 & 9.14 \\
\hline 13 & 4 & 25.00 & 22 & 13.64 & 1435 & 6.83 & 1461 & 6.98 \\
\hline 14 & 9 & 33.33 & 20 & 20.00 & 1497 & 5.61 & 1526 & 5.96 \\
\hline 15 & 2 & 50.00 & 11 & 9.09 & 315 & 2.54 & 328 & 3.05 \\
\hline All GCS scores & 2093 & 58.67 & 1401 & 38.26 & 12,406 & 16.29 & 15,900 & 23.81 \\
\hline
\end{tabular}

Values are from pooled data.

from $31 \%$ to $63 \%$ and on to $79 \%$ as pupil reactivity deteriorated.

\section{Interaction of GCS Score and Pupil Response}

The frequency of loss of pupil reactivity increased with decreasing GCS score: 2.1\% at GCS scores 13 and higher,
$5.5 \%$ at GCS scores $9-12$, and $35.7 \%$ at GCS scores 8 or lower (Tables 2 and 3, Fig. 1). In patients with GCS scores 9-12, unilateral (3.5\%) or bilateral (2.0\%) loss of pupil reactivity occurred at similar rates, but at GCS scores 3-8 loss of reactivity was more often bilateral $(22.2 \%)$ than unilateral (13.5\%).

TABLE 3. Patients with a TBI stratified by GCS score and number of reactive pupils, and the percentage of these patients with unfavorable outcomes 6 months after injury

\begin{tabular}{|c|c|c|c|c|c|c|c|c|}
\hline \multirow[b]{3}{*}{ GCS Score } & \multicolumn{6}{|c|}{ No. of Reactive Pupils } & \multirow{2}{*}{\multicolumn{2}{|c|}{ Total No. of Patients }} \\
\hline & \multicolumn{2}{|r|}{0} & \multicolumn{2}{|c|}{1} & \multicolumn{2}{|r|}{2} & & \\
\hline & $\begin{array}{c}\text { No. of } \\
\text { Patients }\end{array}$ & $\begin{array}{c}\% \text { Unfav Outcome } \\
\text { at } 6 \text { Mos }\end{array}$ & $\begin{array}{c}\text { No. of } \\
\text { Patients }\end{array}$ & $\begin{array}{c}\% \text { Unfav Outcome } \\
\text { at } 6 \text { Mos }\end{array}$ & $\begin{array}{c}\text { No. of } \\
\text { Patients }\end{array}$ & $\begin{array}{c}\% \text { Unfav Outcome } \\
\text { at } 6 \text { Mos }\end{array}$ & $\begin{array}{c}\text { No. of } \\
\text { Patients }\end{array}$ & $\begin{array}{c}\% \text { Unfav Outcome } \\
\text { at } 6 \text { Mos }\end{array}$ \\
\hline 3 & 634 & 89.59 & 178 & 69.66 & 617 & 50.41 & 1429 & 70.19 \\
\hline 4 & 458 & 91.27 & 239 & 82.85 & 462 & 66.02 & 1159 & 79.47 \\
\hline 5 & 322 & 81.68 & 228 & 72.81 & 673 & 58.69 & 1223 & 67.38 \\
\hline 6 & 326 & 70.25 & 259 & 64.86 & 1278 & 45.38 & 1863 & 52.44 \\
\hline 7 & 204 & 45.59 & 208 & 47.12 & 1792 & 36.33 & 2204 & 38.20 \\
\hline 8 & 64 & 60.94 & 111 & 47.75 & 1004 & 32.37 & 1179 & 35.37 \\
\hline 9 & 27 & 51.85 & 46 & 52.17 & 711 & 29.68 & 784 & 31.76 \\
\hline 10 & 23 & 47.83 & 29 & 51.72 & 788 & 28.55 & 840 & 29.88 \\
\hline 11 & 12 & 16.67 & 28 & 39.29 & 836 & 21.53 & 876 & 22.03 \\
\hline 12 & 8 & 25.00 & 22 & 31.82 & 998 & 19.64 & 1028 & 19.94 \\
\hline 13 & 4 & 25.00 & 22 & 27.27 & 1435 & 15.47 & 1461 & 15.67 \\
\hline 14 & 9 & 33.33 & 20 & 40.00 & 1497 & 14.43 & 1526 & 14.88 \\
\hline 15 & 2 & 50.00 & 11 & 9.09 & 315 & 11.75 & 328 & 11.89 \\
\hline All GCS scores & 2093 & 78.55 & 1401 & 62.74 & 12,406 & 31.07 & 15,900 & 40.11 \\
\hline
\end{tabular}

Unfav = unfavorable.

Values are from pooled data. 


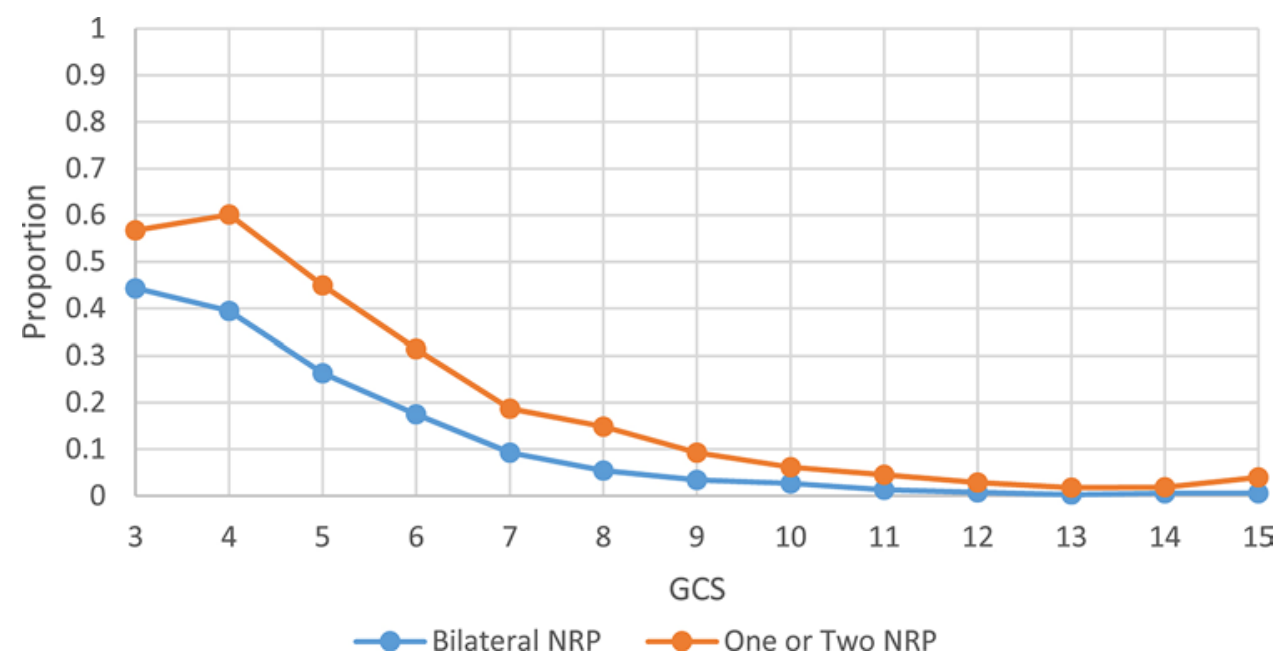

FIG. 1. Variations in the proportions of patients who had pupil abnormalities according to GCS score. NRP = nonreactive pupil(s). Figure is available in color online only.

Loss of pupil reactivity was associated with an increase in mortality rate from $16.3 \%$ when both pupils reacted, to $38.3 \%$ when only 1 reacted, and to $58.7 \%$ when neither pupil reacted. Figure 2 shows that this was not simply a reflection of the association between pupil findings and GCS score, and that the overall pattern (of increasing mortality and unfavorable outcome) was observed across the range of GCS scores. The pattern was most clear and consistent with lower GCS scores; at higher scores there was increased variability, at least in part reflecting the smaller numbers of patients under consideration.

\section{Combined GCS-P and Patient Outcome}

The relationships between the combined GCS-P and the 6-month mortality rate are shown in Table 4 and Fig. 3 left. The combined score extends the range over which differentiation of outcomes is made and increases the highest rate of mortality from $51 \%$ at the lowest GCS score (score 3) to $74 \%$ at GCS-P 1 . The nonmonotonic pattern observed with a higher mortality rate for a GCS score of 4 rather than 3, when only the GCS score was examined, is no longer seen. There is an appearance of "flattening" of the mortality rate at GCS scores 7-10, but the pattern across the full range is in accord with the smooth relationship.

A similar relationship is seen between the GCS-P and the frequency of unfavorable outcome (Table 4 and Fig. 3 right). The highest rate of unfavorable outcome increases from $70 \%$ at GCS score 3 to $90 \%$ at GCS-P 1. Although the observed rate is slightly higher at GCS-P 4 than at GCS-P 3, the confidence intervals overlap and the pattern across the full range is in accord with a smoothly decreasing relationship.

\section{Performance of Methods of Combining Information About GCS Score and Pupil Reactivity}

A comparison of the information content provided by the different ways of analyzing combinations of GCS and pupil findings is shown in Table 5 for the pooled data set. The findings in each of the original data sets is available in Supplementary Table 2a and b.

Nagelkerke's $\mathrm{R}^{2}$ is a measure of the proportion of variability in outcome that is explained by the logistic regression model. All methods of combination are more informative in each data set than the yield from either the GCS
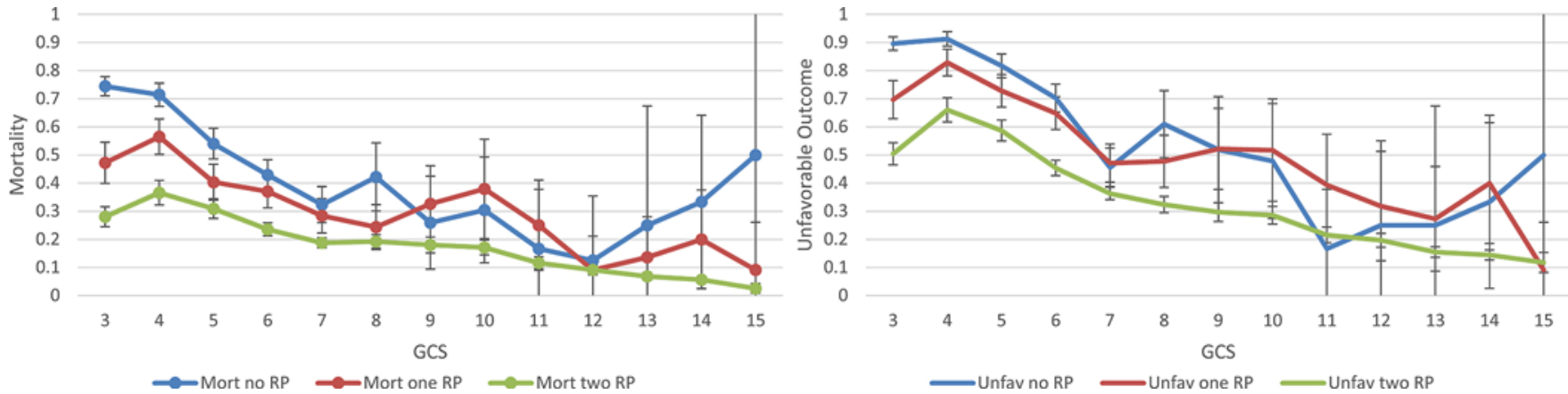

FIG. 2. Mortality (left) and unfavorable outcomes (right) 6 months after head injury according to patient GCS scores at admission. Pooled data were used and patient groups are subdivided according to pupil reactivity. Error bars show $95 \% \mathrm{Cls}$. Mort = mortality: $\mathrm{RP}=$ reactive pupil(s); Unfav = unfavorable outcome. Figure is available in color online only. 
TABLE 4. Relationship of GCS-P to death and unfavorable outcome for the pooled data

\begin{tabular}{crcc}
\hline GCS-P & $\begin{array}{c}\text { No. of } \\
\text { Patients }\end{array}$ & $\begin{array}{c}\text { Death } \\
\text { (\% of patients) }\end{array}$ & $\begin{array}{c}\text { Unfav Outcome } \\
\text { (\% of patients) }\end{array}$ \\
\hline 1 & 634 & 74.45 & 89.59 \\
\hline 2 & 636 & 64.62 & 85.22 \\
\hline 3 & 1178 & 40.92 & 65.53 \\
\hline 5 & 1016 & 39.47 & 68.90 \\
\hline 6 & 1136 & 32.57 & 57.75 \\
\hline 7 & 1550 & 24.97 & 46.26 \\
\hline 8 & 1930 & 19.17 & 37.20 \\
\hline 9 & 1073 & 20.04 & 33.55 \\
\hline 10 & 752 & 18.75 & 30.32 \\
\hline 11 & 824 & 17.35 & 28.88 \\
\hline 12 & 862 & 11.60 & 21.81 \\
\hline 13 & 1029 & 9.43 & 19.92 \\
\hline 14 & 1457 & 7.07 & 15.85 \\
\hline 15 & 1508 & 5.64 & 14.39 \\
\hline All GCS-Ps & 15,900 & 23.81 & 11.75 \\
\hline
\end{tabular}

score or pupil reactivity alone-the latter being the least informative. In contrast, there was very little reduction in performance between the most complex model (model a), in which both the GCS score and the number of nonreacting pupils were treated as separate categorical factors, and the simple arithmetic GCS and pupil reactivity score (GCS-P) model (model f). Thus, in the pooled data for mortality, the $\mathrm{R}^{2}$ for more complex models ranged from $22.7 \%$ to $23.2 \%$ and the $\mathrm{R}^{2}$ for the GCS-P ranged from $21.1 \%$ to $22.6 \%$. Likewise, for unfavorable outcome, the respective values ranged from $26.6 \%$ to $27.6 \%$ and from $25.3 \%$ to $26.6 \%$. The pattern was consistent across the two original data sets (Supplementary Table 2a and b).

\section{Discussion}

GCS score and pupil reaction are the two clinical fea- tures that provide the most information about prognosis for a head-injured patient. ${ }^{4,10,27}$ Our findings demonstrate that combining these features yields more informative data than using either alone, and that a simple arithmetical score, the GCS-P, performs almost as well as more complex methods of integration.

We used the two largest databases on head-injured patients, the CRASH and IMPACT, in which information on early GCS score, pupil findings, and late patient outcome are available for individual patients. The total numbers of patients are similar in the two databases, but there are variations in their composition. The IMPACT database is skewed toward patients with severe head injuries (81\%) compared with the CRASH database (39\%), yet in the CRASH database there are few people with GCS score 15 . We used a pooled data set to compensate for these differences and to increase the generalizability of our findings.

The increase in mortality and unfavorable outcome observed at a GCS score of 4 compared with a GCS score of 3 has been reported in other papers..$^{12,17}$ The reason for this paradoxical finding is unclear. Importantly, though, this finding ceased to exist when the GCS score and pupil data were combined into GCS-Ps of 1-4. A comparison of the effect on patient outcome of the pupil response at each level of the GCS indicates that pupil data provide additional information at low GCS scores, as would be expected, because an abnormal pupil response is more common in patients with severe brain injury and a low GCS score.

A comparison of the fitted linear logistic relationship between patient outcome and GCS-P indicates that a GCS-P of 1 or 2 slightly underestimates the likelihood of adverse outcome. We considered increasing the weighting attached to the loss of pupil reactivity, by assigning a score of -2 when only 1 pupil reacts and a score of -4 when neither pupil reacts; however, we rejected this in favor of using the simpler method. We also considered subtracting the PRS only from GCS scores below a specific threshold of injury severity, for example at a GCS score lower than 8. This too was rejected in favor of simplicity and the finding that, although data for higher GCS scores were more variable in view of the smaller numbers of pa-
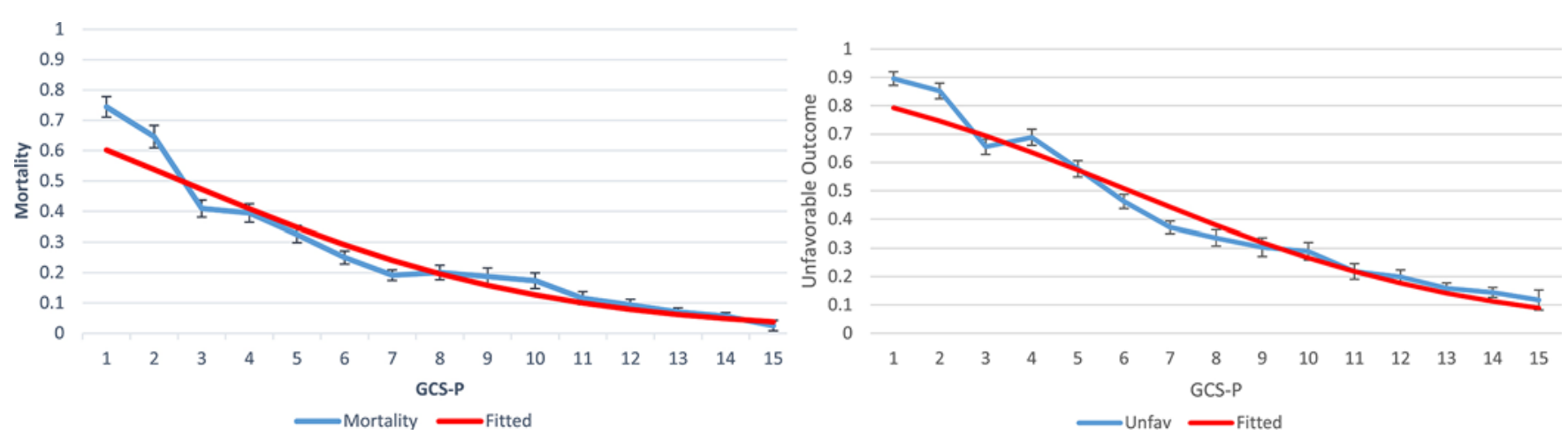

FIG. 3. Mortality 6 months after head injury (left) and unfavorable outcomes (right) according to patient GCS-Ps at admission; the results were determined using pooled CRASH and IMPACT data. Error bars show $95 \% \mathrm{Cls}$. The fitted lines come from logistic regression models in which the GCS-P was treated as a continuous variable. Figure is available in color online only. 
TABLE 5. Predictive yield of different models of combining information about the relationships of GCS score, pupil response, and outcome with respect to mortality and unfavorable outcome in the pooled data from the IMPACT and CRASH databases

\begin{tabular}{lcc}
\hline \multicolumn{1}{c}{ Model } & $\begin{array}{c}\text { Nagelkerke's R (\%) } \\
\text { Modeling Death }\end{array}$ & $\begin{array}{c}\text { Nagelkerke's R } \text { (\%) }^{2} \\
\text { Modeling Unfav Outcome }\end{array}$ \\
\hline GCS score \& pupil reactivity separately as categorical variables (model a) & 23.2 & 27.6 \\
\hline GCS score as linear \& pupil reactivity as categorical variables (model b) & 22.7 & 26.7 \\
\hline GCS score as categorical \& pupil reactivity as linear variables (model c) & 23.2 & 27.6 \\
\hline GCS score \& pupil reactivity as separate linear variables (model d) & 22.7 & 26.6 \\
\hline GCS-P alone as categorical variable (model e) & 22.6 & 26.6 \\
\hline GCS-P alone as linear variable (model f) & 21.1 & 25.3 \\
\hline GCS score alone as categorical variable & 19.2 & 24.4 \\
\hline GCS score alone as linear variable & 18.3 & 22.8 \\
\hline Pupil reactivity alone as categorical variable & 15.4 & 16.1 \\
\hline Pupil reactivity alone as linear variable & 15.3 & 15.9 \\
\hline
\end{tabular}

Nagelkerke's $\mathrm{R}^{2}$ is a measure of the proportion of variability in outcome that is "explained" by the logistic regression model.

tients studied, there was a relationship across the whole range.

Using GCS scores to classify injuries as mild (GCS scores 13-15), moderate (GCS scores 9-12), or severe (GCS scores $\leq 8$ ) is widely used and was applied in our pooled data set to $20.8 \%, 22.2 \%$, and $57.0 \%$ of patients, respectively. ${ }^{32}$ After application of the GCS-P, these proportions were altered only slightly to $20.6 \%, 21.8 \%$, and $57.6 \%$ of patients. The very similar allocation of patients to the severe injury category resulted because the finding of 1 or 2 nonreacting pupils occurs mainly in patients with low GCS scores; there is thus not a big difference between a GCS cut-off score of 8 or lower and a GCS-P cut-off score of 8 or lower. Using the GCS-P, the existing classification therefore remains valid, although the score separation within the severe injury group is increased from 6 to 8 categories. Nevertheless, the full clinical picture should be taken into account in determining how a patient's case should best be managed. ${ }^{13,24}$

Other methods of adding information to the Glasgow Coma Scale or its components to extend its spectrum have been described. One version aimed to expand discrimination in the large number of patients with mild head injuries by using additional information about memory. ${ }^{16}$ Most other proposals focused on patients with severe brain injury, adding a variety of so-called brainstem features. The Glasgow-Liège score includes pupil response, the oculocephalic reflex, and the oculocardiac reflex. ${ }^{3}$ The Innsbruck Coma Scale adds pupil size, pupil response to light, eyeball position and movements, and oral automatisms in a 31-point assessment. ${ }^{2}$ The FOUR (Full Outline of UnResponsiveness) score adds pupil, corneal, and cough reflexes, myoclonus, and specific breathing patterns. ${ }^{32}$ Surprisingly, in studies in which predictive performance has been compared, the extra prognostic yield above the GCS score provided by these additional sources usually has not been significant and, with the exception of pupil response, the value of their contributions have been questioned. ${ }^{6}$ Where a difference has been observed, it has usually been that the lowest points in the composite scores are associated with higher rates of mortality than those seen at GCS score 3, and our findings in the present study show that is achieved using the GCS-P. ${ }^{18}$

The simple addition of pupil information to the GCS score increases the likelihood of mortality in a patient with a GCS score of 3 from $51 \%$ to $74 \%$ and the likelihood of unfavorable outcome from $70 \%$ to $90 \%$ when the GCS-P is 1 . This suggests that more complex methods of incorporating an assessment of brainstem function may be unnecessary. More prospective studies with other data sets, such as CENTER-TBI (Collaborative European NeuroTrauma Effectiveness Research in TBI, https://www.center-tbi.eu/) and TRACK-TBI (Transforming Research and Clinical Knowledge in Traumatic Brain Injury, https://tracktbi.ucsf. edu/), are desirable to further characterize the GCS-P and to compare its performance with more elaborate composite scores. ${ }^{9}$

The combined GCS-P is not intended to replace the role of separate assessment and reporting of each component of the Glasgow Coma Scale and pupil response in the care of individual patients. This remains the most informative way of determining and sharing a "picture" of the patient's condition and how it may be changing. ${ }^{29}$ When the various components of the Glasgow Coma Scale are combined there is some loss of information..$^{21,30}$ This has been accepted as a trade-off in the interests of simplicity and the utility of the total GCS score as a "shorthand summary." The GCS-P retains this simplicity while expanding information about the severity of a patient's clinical state and prognosis. The role of the GCS-P now needs assessment and evaluation by the clinical community. We expect that this summary score will be especially valuable in cases of severe head injury and will prove useful in drawing up guidelines and describing findings in groups of patients. Neither the GCS-P nor, indeed, any clinical assessment tool can determine pathophysiological mechanisms; identifying these mechanisms requires further investigations. However, the GCS-P can provide a guide to performing such investigations, to repeating them, and to the success of targeted interventions. Prediction of outcome should not be used alone in management decisions.

Prognosis, in addition to clinical responsiveness, is in- 
fluenced by several factors. In addition to the importance of the patient's age, these include findings of imaging and other examinations, the presence of extracranial injuries, and systemic insults. ${ }^{27}$ Multivariate models, such as those developed using data from the $\mathrm{CRASH}^{19}$ and IMPACT ${ }^{27}$ databases, provide the most rigorous approach to collect this information to provide an estimate of the probability of a patient's outcome. Nevertheless, such formal models cannot be used to predict with certainty the clinical course of a head-injured patient; all leave a substantial amount of individual variability in outcome unexplained and have found, at most, a limited place in clinical practice. ${ }^{8}$ As a consequence, although clinicians identify prognosis as a factor in their decision making, the prognosis remains exposed to personal subjective opinions. ${ }^{1,11}$ By providing a simple but informative index, the GCS-P may be useful in avoiding biases among clinicians about patient prognosis.

Perel et al. advised that for prognostic models to be clinically useful they should be presented in a user-friendly way to be easily applied in the clinical scenario. ${ }^{20}$ The GCS-P already brings together the two most important clinical predictive features. How this assessment tool can be built upon to add other predictive features in a graphic presentation is described in our companion paper..$^{14}$

\section{Acknowledgments}

We thank Professor Andrew Maas, University of Antwerp, and Professor Ian Roberts, London School of Hygiene and Tropical Medicine, for allowing the use of information from the IMPACT and CRASH databases, respectively.

\section{References}

1. Barlow P, Teasdale G: Prediction of outcome and the management of severe head injuries: the attitudes of neurosurgeons. Neurosurgery 19:989-991, 1986

2. Benzer A, Mitterschiffthaler G, Marosi M, Luef G, Pühringer F, De La Renotiere K, et al: Prediction of non-survival after trauma: Innsbruck Coma Scale. Lancet 338:977-978, 1991

3. Born JD: The Glasgow-Liège Scale. Prognostic value and evolution of motor response and brain stem reflexes after severe head injury. Acta Neurochir (Wien) 91:1-11, 1988

4. Braakman R, Gelpke GJ, Habbema JD, Maas AI, Minderhoud JM: Systematic selection of prognostic features in patients with severe head injury. Neurosurgery 6:362-370, 1980

5. Brain Trauma Foundation, American Association of Neurological Surgeons: Management and Prognosis of Severe Traumatic Brain Injury. Campbell, CA: Brain Trauma Foundation, 2000

6. Eken C, Kartal M, Bacanli A, Eray O: Comparison of the Full Outline of Unresponsiveness Score Coma Scale and the Glasgow Coma Scale in an emergency setting population. Eur J Emerg Med 16:29-36, 2009

7. Jennett B, Teasdale G, Braakman R, Minderhoud J, KnillJones R: Predicting outcome in individual patients after severe head injury. Lancet 1(7968):1031-1034, 1976

8. Latronico N: Prediction is very difficult, especially about the future. Crit Care Med 43:505-506, 2015

9. Maas AIR, Menon DK, Steyerberg EW, Citerio G, Lecky F, Manley GT, et al: Collaborative European NeuroTrauma Effectiveness Research in Traumatic Brain Injury (CENTERTBI): a prospective longitudinal observational study. Neurosurgery 76:67-80, 2015

10. Marmarou A, Lu J, Butcher I, McHugh GS, Mushkudiani
NA, Murray GD, et al: IMPACT database of traumatic brain injury: design and description. J Neurotrauma 24:239-250, 2007

11. Moore NA, Brennan PM, Baillie JK: Wide variation and systematic bias in expert clinicians' perceptions of prognosis following brain injury. Br J Neurosurg 27:340-343, 2013

12. Moskopp D, Stähle C, Wassmann H: Problems of the Glasgow Coma Scale with early intubated patients. Neurosurg Rev 18:253-257, 1995

13. Moulton C, Pennycook AG: Relation between Glasgow coma score and cough reflex. Lancet 343:1261-1262, 1994

14. Murray GD, Brennan PM, Teasdale GM: Simplifying the use of prognostic information in traumatic brain injury. Part 2: Graphical presentation of probabilities. J Neurosurg [epub ahead of print April 10, 2018; DOI: 10.3171/2017.12. JNS172782]

15. Nagelkerke NJD: A note on a general definition of the coefficient of determination. Biometrika 78:691-692, 1991

16. Nell V, Yates DW, Kruger J: An extended Glasgow Coma Scale (GCS-E) with enhanced sensitivity to mild brain injury. Arch Phys Med Rehabil 81:614-617, 2000

17. Osler T, Cook A, Glance LG, Lecky F, Bouamra O, Garrett $\mathrm{M}$, et al: The differential mortality of Glasgow Coma Score in patients with and without head injury. Injury 47:18791885,2016

18. Peng J, Deng Y, Chen F, Zhang X, Wang X, Zhou Y, et al: Validation of the Chinese version of the FOUR score in the assessment of neurosurgical patients with different level of consciousness. BMC Neurol 15:254, 2015

19. Perel P, Arango M, Clayton T, Edwards P, Komolafe E, Poccock S, et al: Predicting outcome after traumatic brain injury: practical prognostic models based on large cohort of international patients. BMJ 336:425-429, 2008

20. Perel P, Edwards P, Wentz R, Roberts I: Systematic review of prognostic models in traumatic brain injury. BMC Med Inform Decis Mak 6:38, 2006

21. Reith FCM, Lingsma HF, Gabbe BJ, Lecky FE, Roberts I, Maas AIR: Differential effects of the Glasgow Coma Scale Score and its Components: an analysis of 54,069 patients with traumatic brain injury. Injury 48:1932-1943, 2017

22. Reith FCM, Van den Brande R, Synnot A, Gruen R, Maas AIR: The reliability of the Glasgow Coma Scale: a systematic review. Intensive Care Med 42:3-15, 2016

23. Roberts I, Yates D, Sandercock P, Farrell B, Wasserberg J, Lomas G, et al: Effect of intravenous corticosteroids on death within 14 days in 10008 adults with clinically significant head injury (MRC CRASH trial): randomised placebo-controlled trial. Lancet 364:1321-1328, 2004

24. Rotheray KR, Cheung PSY, Cheung CSK, Wai AKC, Chan DYS, Rainer TH, et al: What is the relationship between the Glasgow coma scale and airway protective reflexes in the Chinese population? Resuscitation 83:86-89, 2012

25. Royston P, Moons KGM, Altman DG, Vergouwe Y: Prognosis and prognostic research: developing a prognostic model. BMJ 338:b604, 2009

26. Steyerberg EW, Moons KGM, van der Windt DA, Hayden JA, Perel P, Schroter S, et al: Prognosis Research Strategy (PROGRESS) 3: prognostic model research. PLoS Med 10:e1001381, 2013

27. Steyerberg EW, Mushkudiani N, Perel P, Butcher I, Lu J, McHugh GS, et al: Predicting outcome after traumatic brain injury: development and international validation of prognostic scores based on admission characteristics. PLoS Med 5:e165, 2008

28. Teasdale G, Jennett B, Murray L, Murray G: Glasgow coma scale: to sum or not to sum? Lancet 2(8351):678, 1983

29. Teasdale G, Maas A, Lecky F, Manley G, Stocchetti N, Murray G: The Glasgow Coma Scale at 40 years: standing the test of time. Lancet Neurol 13:844-854, 2014 
30. Teasdale G, Murray G, Parker L, Jennett B: Adding up the Glasgow Coma Score. Acta Neurochir Suppl (Wien) 28:1316,1979

31. Teasdale G, Jennett B: Assessment of coma and impaired consciousness. A practical scale. Lancet 2:81-84, 1974

32. Wijdicks EFM, Bamlet WR, Maramattom BV, Manno EM, McClelland RL: Validation of a new coma scale: The FOUR score. Ann Neurol 58:585-593, 2005

\section{Disclosures}

Financial support for this study was provided by the Muriel Cooke Bequest to the University of Glasgow.

\section{Author Contributions}

Conception and design: all authors. Acquisition of data: Murray, Teasdale. Analysis and interpretation of data: all authors. Drafting the article: all authors. Critically revising the article: all authors. Reviewed submitted version of manuscript: all authors. Approved the final version of the manuscript on behalf of all authors: Brennan. Statistical analysis: Murray. Administrative/technical/material support: Brennan.

\section{Supplemental Information}

Further information on the GCS and GCS-P is available at www. glasgowcomascale.org.

\section{Online-Only Content}

Supplemental material is available with the online version of the article.

Supplementary Tables 1 and 2. https://thejns.org/doi/suppl/10. 3171/2017.12.JNS172780.

\section{Companion Papers}

Murray GD, Brennan PM, Teasdale GM: Simplifying the use of prognostic information in traumatic brain injury. Part 2: Graphical presentation of probabilities. DOI: 10.3171/2017.12.JNS172782.

\section{Previous Presentations}

Portions of this work were presented in poster form at the International Neurotrauma Symposium in 2016 and the Society of British Neurosurgeons Spring Plenary meeting in 2016.

\section{Correspondence}

Paul Brennan: Centre for Clinical Brain Sciences, University of Edinburgh, United Kingdom. paul.brennan@ed.ac.uk. 